\title{
Invasive Pneumococcal Disease Case Series in Pediatric Inpatients: A Clinical Experience from South India
}

\author{
Rajagopal Kishore Kumar ${ }^{1}$, Sanjay Swamy ${ }^{2}$, Adithi Shetty ${ }^{3}$
}

\begin{abstract}
Aim and objective: To monitor the causative pathogens, serotype distribution, and antimicrobial resistance patterns for pediatric invasive pneumococcal disease (IPD).

Background: In India, IPD is a major contributing factor to the high under-five mortality rate, but surveillance-based studies are scarce on the subject, although vaccines are available for prevention.

Case descriptions: We present a case series of IPD among Indian children aged $\leq 12$ years attending a pediatric specialty hospital in Bengaluru, South India, over 5 years. Nine IPD cases were seen between June 2014 and July 2019 including both immunized and unimmunized children. The clinical presentation included pneumonia that progressed to sepsis, empyema, and pleural effusion in seven children and febrile seizures in one patient. Streptococcus pneumoniae was isolated from all the blood and pleural fluid cultures. The serotypes isolated were 19F, 19A, 2, $14,9 \mathrm{~V} / \mathrm{A}, 6 \mathrm{~A} / 6 \mathrm{~B}$, and 3, which were all vaccine-preventable. Three cases were susceptible to all antibiotics, while others showed antimicrobial resistance to cotrimoxazole, benzylpenicillin, erythromycin, and clindamycin. All but one patient recovered uneventfully.

Conclusion: Continued surveillance for IPDs and serotyping of pneumococcal isolates is important to study the effects of vaccination and monitor the emergence of non-vaccine-preventable serotypes.

Clinical significance: Although IPD-causing pneumococcal serotypes have been documented in the Indian scenario, data are scarce on the emergence of non-vaccine-preventable serotypes, particularly in Indian children with IPD. Since the availability of pneumococcal vaccine (PCV) in India, this is possibly the first case series of IPD in Indian immunized children. The case series reveals that IPD-causing pneumococcal serotypes in Indian children are mostly vaccine-preventable and susceptible to standard antibiotics.
\end{abstract}

Keywords: Invasive pneumococcal disease, Pediatric, Serotype, Vaccine.

Pediatric Infectious Disease (2022): 10.5005/jp-journals-10081-1293

\section{BACKGROUND}

India is ranked among the top 10 countries with the highest mortality among children aged below 5 years, with pneumonia being the most common cause. ${ }^{1}$ Streptococcus pneumoniae is one of the most common bacterial isolates from pneumonia, accounting for $13-40 \%$ of these cases. ${ }^{1}$ India contributes to the largest number of pneumococcal disease-associated deaths among children aged 1-59 months. ${ }^{1}$ Besides, meningitis is another clinical presentation of pneumococcal disease, accounting for high mortality in children below 5 years of age. ${ }^{1}$

Invasive pneumococcal disease (IPD), defined as the isolation of pneumococci from normally sterile sites of the body, mostly affects children $<2$ years old. ${ }^{2}$ Invasive pneumococcal diseases are mostly fatal, with the rate of fatality ranging from 11 to $60 \%{ }^{3}$ The incidence of IPD in developing countries like India is several times higher than that in industrialized countries, owing to genetic factors and socioeconomic differences. ${ }^{2}$ However, data for IPDs are scarce in low-income countries due to multiple causes, with pre-hospitalization use of antibiotics among children being the predominant cause. Other important causes are suboptimal specimen collection, and inadequate laboratory facilities, which lead to inconsistent data. ${ }^{3}$

To date, $>90$ serotypes of pneumococcus have been identified among which 23 serotypes cause the majority of IPD. Ten major serotypes cause $62 \%$ of the IPD cases worldwide. ${ }^{2}$ Various vaccines are available to prevent pneumococcal infections. ${ }^{4}$ These vaccines include pneumococcal conjugate vaccine (PCV-7), which targets serotypes 4, 6B, 9V, 14, 18C, 19F, and 23F; in addition to these
${ }^{1}$ Department of Neonatology, Cloudnine Hospitals, Bengaluru, Karnataka, India

${ }^{2,3}$ Department of Pediatrics, Cloudnine Hospitals, Bengaluru, Karnataka, India

Corresponding Author: Rajagopal Kishore Kumar, Department of Neonatology, Cloudnine Hospitals, Bengaluru, Karnataka, India, Phone: +91 9900155001, e-mail: drkishore@cloudninecare.com

How to cite this article: Kumar RK, Swamy S, Shetty A. Invasive Pneumococcal Disease Case Series in Pediatric Inpatients: A Clinical Experience from South India. Pediatr Inf Dis 2022;4(1):1-5.

Source of support: The study was funded by Cloudnine (Kids Clinic India Pty Ltd.) research fund.

Conflict of interest: None

serotypes, PCV-10 targets 1, 5 and 7F, and PCV-13 targets 3, 6A, and $19 \mathrm{~A}$ serotypes. ${ }^{5}$

For the effective implementation of a PCV, awareness of regionspecific serotypes is of utmost importance, which facilitates a more appropriate selection of vaccines. However, data are scarce on serotypes of Indian pneumococcal strains. ${ }^{4}$ This is largely due to challenges associated with the isolation and accurate identification of pneumococcal strains from clinical specimens. Although simple and efficient methods of serotyping are available in India, like a polymerase chain reaction, their implementation in Indian laboratories is still limited. ${ }^{2}$

Although in the recent past, several multicenter studies by surveillance groups have documented a substantial burden of pneumococcal disease in Indian children, along with their serotype 
epidemiology, these data do not accurately reflect current trends and lack region-wise data in India. Therefore, they might also underestimate the actual burden of IPD among Indian children. The unavailability of the latest, region-wise trends of the prevailing pneumococcal serotypes in India necessitates the need to bridge these gaps, which will subsequently aid in determining the suitable vaccine for appropriate selection. ${ }^{3}$

With this background, herein, we present a case series of IPDs in nine children attending the pediatric specialty hospital in South India, to identify the prevailing serotypes and immunization status of children against pneumococcal diseases in this part of the country. The present case series of IPD involved children aged $\leq 12$ years who attended a pediatric specialty hospital in Bengaluru, South India, over 5 years from June 2014 to July 2019, including both immunized and unimmunized children. Patients with suspected IPD were investigated and treated according to standard protocol, including antibiotics when required. The background history of respiratory diseases and vaccination status of the children were recorded at presentation. Clinical features recorded for most of the cases included the presence and duration of fever, chills, cough and cold, hurried breathing, respiratory distress, and chest and abdominal pain. Initial vital signs recorded were heart rate, respiratory rate, peripheral capillary oxygen saturation $\left(\mathrm{SpO}_{2}\right)$, hemoglobin, C-reactive protein (CRP), total leukocyte count, and platelet count. If required, patients were provided ventilation or pediatric intensive care unit (PICU) support. Chest radiographs were obtained in all children with clinically suspected pneumonia.

Additional investigations included a collection of pleural fluid and blood samples under sterile conditions; the samples were cultured on $5 \%$ sheep blood agar and MacConkey agar. Colonies showing alpha-hemolysis on blood agar and chocolate agar under $10 \% \mathrm{CO}_{2}$ and no growth on MacConkey agar were selected for identification using VITEK ${ }^{\circledast} 2$ GP ID cards (from bioMérieux) after testing for bile solubility and optochin sensitivity. Antibiotic sensitivity testing was performed using VITEK 2 AST cards. Although not a routine method, S. pneumoniae isolates from these nine cases were serotyped, and hence these cases were selected for this case series. The serotyping for this small number of cases was done free of cost by the World Health Organization (WHO) accredited lab in Kempegowda Institute of Medical Sciences (KIMS), Bengaluru, on our request.

Serotyping was done by coagglutination with Neufeld antisera test; Pool A-I, 25 types and 21 groups corresponding with the specific set of factor sera were obtained from Statens Serum Institut (Copenhagen, Denmark) at another center (Central Research Laboratory KIMS Hospital and Research Centre, Bengaluru). Furthermore, quantitative multiplex polymerase chain reaction (QmPCR) was used for serotyping.

Data were collected from the medical records of the patients stored in the microbiological and radiological databases of the hospital. The study was approved by the institutional ethics committee. Since this was a retrospective study, the requirement of informed consent from individual patients was waived. As this is a case series involving only nine patients, detailed statistical analysis was not performed.

\section{Case Descriptions}

\section{Case 1}

A 6-year-old male child presented to pediatric emergency with complaints of fever with chills, cough, and cold for 4 days progressing to hurried breathing. The patient was not immunized with the PCV; developmental history was appropriate for age. No significant past and family histories were recorded. Upon examination, nasal flaring, chest retraction, and cold peripheries were noted. Examination of the vitals showed a heart rate of 170/ minute, respiratory rate of 72 counts/minute, and BP of $98 / 70 \mathrm{~mm}$ Hg. On auscultation, bronchial breath sounds were noted in the left axillary and infrascapular areas. Examination of the central nervous system (CNS) showed the patient to be drowsy but well oriented and interacting. No significant findings were observed per abdomen and in the cardiovascular system (CVS). A diagnosis of left-sided pneumonia with sepsis was made, and the patient was intubated and mechanically ventilated. Blood for culture tests and other investigations were drawn before initiation of intravenous (IV) meropenem and vancomycin, as per the antibiotic policy of the unit for community-acquired pneumonia (CAP). Within 2 hours, the patient was found to be unresponsive to verbal commands with cold peripheries, non-palpable pulse, and mildly dilated pupils. Furthermore, the endotracheal secretions were hemorrhagic, and fresh frozen plasma was transfused. Resuscitation was initiated, but the patient failed to revive. The patient was declared dead about 12 hours after admission. Blood culture results available after 72 hours showed the growth of S. pneumoniae serotype 19F, non-susceptible to benzylpenicillin (meningitis interpretation), erythromycin, clindamycin, and cotrimoxazole but moderately susceptible to ceftriaxone (meningitis interpretation).

\section{Case 2}

A male toddler aged 2 years and 1 month presented to the pediatric emergency with complaints of hurried breathing and grunting for 1 day, fever with chills for 4 days along with cough, and cold for the past 1 week. The patient was immunized with PCV (Prevenar13), and the developmental history was appropriate for age. No significant past and family histories were recorded. The heart rate of 154/ minute, respiratory rate of $50 \mathrm{bpm}$, and saturation of peripheral oxygen $\left(\mathrm{SPO}_{2}\right) 88 \%$ in room air were recorded. Severe grunting and suprasternal and bilateral intercostal recession were noted. There was a dull note in percussion and auscultation revealed reduced air entry in the right lower zone. No significant findings were observed per abdomen, CNS, and CVS. A diagnosis of pneumonia with right-sided pleural effusion was made. Chest radiographs showed haziness in the right lower zone, obscuring the right costopleural angle, hemidiaphragm, and the cardiac border. Other investigations included hemoglobin $9.8 \mathrm{~g} \%$, total leukocyte count $2,500 / \mathrm{cm}^{3}$, neutrophils $38 \%$, lymphocytes $58 \%$, platelet 2.8 lacs/ $\mathrm{mm}^{3}$, and CRP $121.6 \mathrm{mg} / \mathrm{dL}$. Intravenous amikacin and piperacillintazobactam were initiated. Streptococcus pneumoniae serotype 19A, non-susceptible to benzylpenicillin (meningitis interpretation) and erythromycin but moderately susceptible to cotrimoxazole, was isolated from the pleural fluid. The toddler recovered uneventfully.

\section{Case 3}

A 3-year-old female child presented with complaints of hurried breathing for 1 day, fever with chills for 2 days, and cough, cold, and earache for 4 days. The patient presented with a history of the right bronchopleural fistula with a chest tube in situ. She was not immunized with the PCV, and her developmental history was appropriate for age. No significant family history was given. On examination, heart rate of 140 /minute, respiratory rate of $50 \mathrm{cpm}$, and $\mathrm{SPO}_{2} 88 \%$ in room air were recorded. Respiratory system examination showed reduced air entry with a dull note 
on percussion and crackles on the right side. Blood investigations revealed total leukocyte count of $14,100 / \mathrm{mm}^{3}$, neutrophils $79 \%$, lymphocytes $16 \%$, platelet $2.7 \mathrm{lacs} / \mathrm{cm}^{3}$, and CRP of $103.9 \mathrm{mg} /$ $\mathrm{dL}$. The patient was started on IV amikacin and amoxicillinclavulanic acid. Culture examination revealed S. pneumoniae serotype 14 susceptible to penicillin, ceftriaxone, levofloxacin, and cotrimoxazole. The infant recovered uneventfully.

\section{Case 4}

A male infant aged 2 months and 28 days was brought with complaints of fever for 2 days and hurried to breathe for 1 day. The patient was not immunized with the PCV; developmental history was appropriate for age with no significant family history. Examination of vitals revealed a heart rate of 156 /minute, respiratory rate of $70 \mathrm{cpm}$, and $\mathrm{SPO}_{2} 92 \%$ in room air were recorded. The child was admitted to the pediatric intensive care unit (PICU) with severe respiratory distress with stridor and irritability. He was intubated in view of persisting respiratory distress, with no response to nebulization. As the patient had tense anterior fontanelle, suggestive of pyogenic meningitis, a lumbar puncture was done to remove a sample of cerebrospinal fluid (CSF) that was suggestive of pyogenic meningitis. The CSF PCR was positive for S. pneumonia. The patient was treated with IV ceftriaxone and IV vancomycin in meningitic doses. Blood culture yielded S. pneumoniae serotype 2 susceptible to all antibiotics. The infant recovered uneventfully.

\section{Case 5}

A female patient aged 5.7 years was brought to the emergency with complaints of cough and cold for 5 days, fever for 3 days, severe breathing difficulty, and abdominal pain. The child had a history of a blocked nose with difficulty in nasal breathing. The patient had no significant past and family histories. Examination of vitals revealed a heart rate of 158/minute, respiratory rate of 68 $\mathrm{cpm}$, and $\mathrm{SPO}_{2} 80 \%$ in room air. Respiratory system examination revealed reduced air entry over the left chest with no adventitious sounds. A diagnosis of left-sided pneumonia with effusion was made. The child was clinically suspected to have severe sepsis with septic shock and treated as per the standard protocol. Initial blood investigations revealed low white blood cell (WBC) count and high CRP $(>20)$. The results of blood culture were inconclusive serum (quantitative real-time) PCR showed the presence of $S$. pneumoniae. Chest radiographs revealed a left-sided white-out lung (Fig. 1). Ultrasonography (USG) of the chest revealed diffuse consolidation of the left lung with pleural effusion. The pleural fluid analysis revealed features of empyema. Pleural fluid PCR revealed S. pneumoniae with $19 \mathrm{~F}$ serotype. The child recovered uneventfully after 3 weeks of IV antibiotic treatment.

\section{Case 6}

A female patient aged 3 years presented with continuous cough and fever for 12 hours of hospital discharge the previous day. The patient initially had right-sided pneumonia and was discharged from the hospital just the previous day. Vitals revealed a heart rate of $28 /$ minute and a respiratory rate of $38 \mathrm{cpm}$. The chest $\mathrm{X}$-ray revealed right-sided pneumonia (Fig. 1); serum (quantitative real-time) PCR showed the presence of S. pneumoniae and a $19 \mathrm{~F}$ serotype was identified. The child was administered IV antibiotics and fed orally. For 24 hours, the child was afebrile, with reduced cough, hemodynamically stable, and tolerating feeds and taking medications well. After 24 hours, the child was discharged in stable condition.

\section{Case 7}

A female child aged 12 years was presented with respiratory distress, severe cough, and lethargy. The patient had a cough for 3 days, initially dry but later turned productive (and blood-tinged) with high-grade intermittent fever. Examination of vitals revealed a heart rate of $170 /$ minute, respiratory rate of $50 \mathrm{cpm}$, and $\mathrm{SPO}_{2} 88 \%$ in room air. Chest radiograph was suggestive of bilateral pneumonia with collapse/consolidation of right upper lobe (Fig. 1). Chest USG showed the collapse of the right lobe with $58-70 \mathrm{~cm}^{3}$ of pleural effusion on the right side. Streptococcus pneumoniae serotype $9 \mathrm{~V} / \mathrm{A}$ susceptible to ceftriaxone, vancomycin, and clindamycin was isolated from the pleural fluid. The child recovered uneventfully in 2 weeks.

\section{Case 8}

A male child aged 6.9 years was brought to the emergency service with complaints of respiratory distress for a day. Further elucidating the complaints, the parents gave a history of cough and fever for 7 days. The child had been vaccinated with 3 doses of PCV 10 by a local pediatrician. There was no significant past and family history. All the vitals were recorded (heart rate of 150 /minute, respiratory rate of $60 \mathrm{cpm}, \mathrm{SPO}_{2} 86-88 \%$ in room air). Chest radiographs revealed a right-sided white-out lung, suggestive of right-sided pneumonia with pleural effusion (Fig. 1). Ultrasonography revealed massive pleural effusion. The pleural fluid analysis revealed features suggestive of empyema. The child also developed features of acute respiratory distress syndrome (ARDS) and septic shock. Serum PCR of pleural fluid revealed S. pneumoniae of serotype $6 \mathrm{~A} / 6 \mathrm{~B}$ responsive to ceftriaxone, vancomycin, and clindamycin. The child recovered after 2 weeks.

\section{Case 9}

A male patient aged 2.9 years was brought to a hospital with complaints of moderate-to-high-grade fever, cough, and running nose for 5 days, with respiratory distress. All the vitals were recorded (heart rate of 120/minute, respiratory rate of $46 \mathrm{cpm}$, and $\mathrm{SPO}_{2} 86-88 \%$ in room air) were recorded. Blood examination revealed elevated CRP levels, leukopenia, and thrombocytopenia with anemia [hemoglobin $(\mathrm{Hb})$ level: $7.7 \%]$. Chest radiographs revealed left-sided pneumonia with pleural effusion. The child was diagnosed with pneumococcal pneumonia with sepsis and complicated pneumonia-left-sided empyema. Streptococcus pneumoniae of serotype 3 was identified. The child recovered uneventfully.

\section{Discussion}

To the best of our knowledge, this is one of the first case series describing IPD disease patterns in hospitalized Indian children, including the $S$. pneumoniae serotypes involved, the clinical presentation of the disease, vaccination status, antimicrobial resistance, and case-fatality rates. In the present case series, $S$. pneumoniae was identified to be the causative pathogen in all the IPD cases. All the children were aged between 2 months and 12 years, and most of them were previously vaccinated with PCVs. The clinical presentation was largely similar involving fever, cough, and cold, with respiratory distress in the majority of the cases. The disease involved pleural effusion in $>50 \%$ of cases and empyema in $\sim 20 \%$ of cases.

The serotypes observed in our study were 19F, 19A, 14, 2, 9V/A, $6 \mathrm{~A} / 6 \mathrm{~B}$, and 3 . An Indian single-center study from South India on 

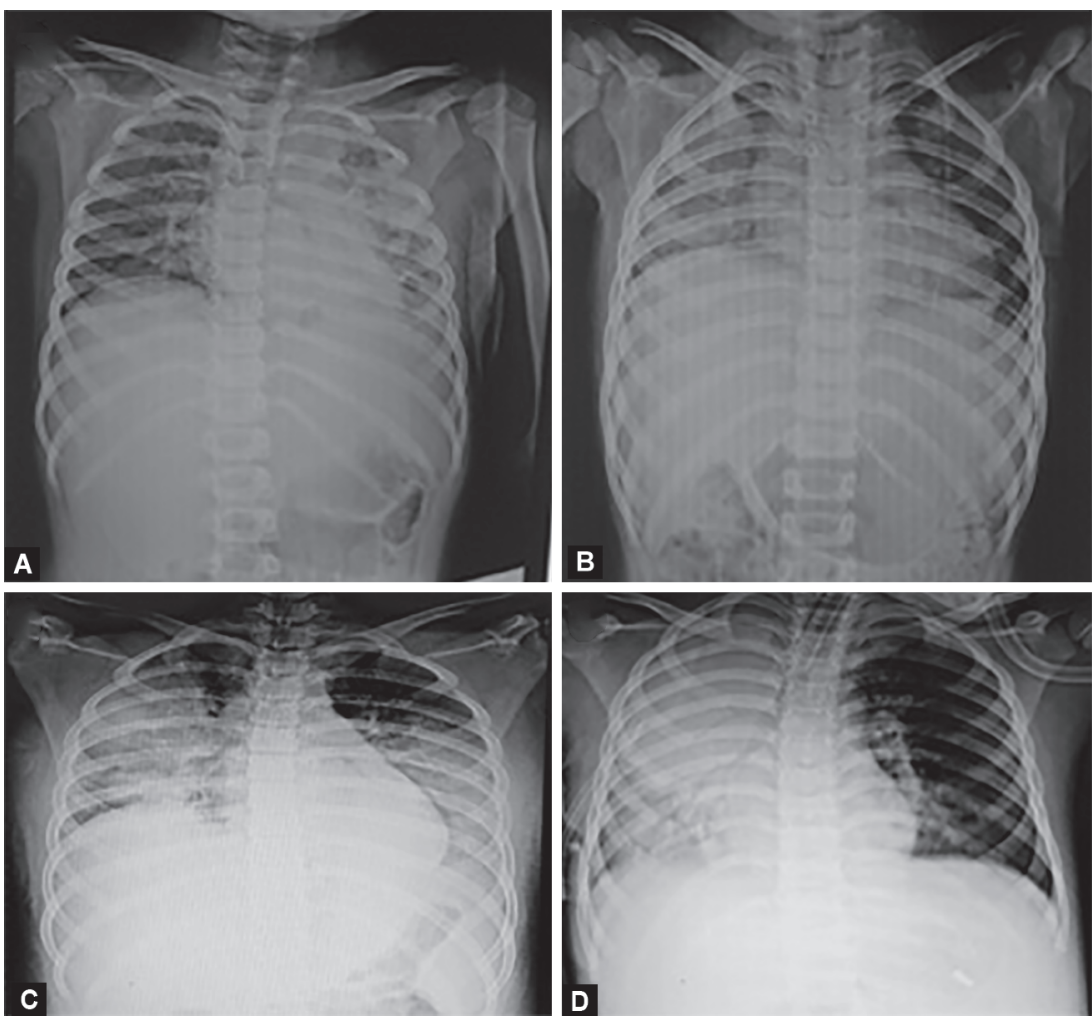

Figs $1 \mathrm{~A}$ to D: Chest radiograph images of pediatric pneumonia cases. (A) Case 5: A 5-year and 7-month-old female child showing left-sided white-out lung; (B) Case 6: A 3-year-old female child showing right-sided pneumonia; (C) Case 7: A 12-year-old female child showing bilateral pneumonia with collapse/consolidation of the right upper lobe; (D) Case 8: A 6-year and 9-month-old male child showing right-sided white-out lung, with pleural effusion

children aged below 5 years reported 14, 19F, 5, 6A, and $6 \mathrm{~B}$ to be the most common pneumococcal serotypes accounting for IPD. ${ }^{5}$ A similar study in South India involving both children and adults found 1, 5 19F, 6B, 14, and 3 to be the most common serotypes isolated from the study population. ${ }^{6}$ An earlier IPD prevalence study in southern India observed that serotypes 5, 6, and 7 contributed to more than one-third of the pneumococcal cases in children. ${ }^{7}$ A large hospital-based surveillance study from South Bengaluru, India identified the following serotypes in children aged 28 days to $<60$ months: $6 \mathrm{~A}, 14,5,6 \mathrm{~B}, 1,18 \mathrm{C}, 19 \mathrm{~A}, 9 \mathrm{~V}, 3,4,10 \mathrm{C}, 18 \mathrm{~A}, 18 \mathrm{~F}$, and $19 \mathrm{~F}^{8}$ Another large hospital-based surveillance study involving 18 hospitals identified $14,1,5$, and $19 \mathrm{~F}$ to be the most common serotypes accounting for IPD in Indian children below 5 years. ${ }^{3} \mathrm{~A}$ systematic review of Indian hospital-based observational studies reported $14,1,19 \mathrm{~F}, 6 \mathrm{~B}, 5,6 \mathrm{~A}, 9 \mathrm{~V}$, and $23 \mathrm{~F}$ to be the most frequent pneumococcal serotypes in children aged $\leq 5$ years. The most common serotypes observed across different geographic regions studied were 14 and $19 A .{ }^{9}$ Therefore, the pneumococcal serotypes observed in the present case series are consistent with other similar reports from India, particularly the southern region. However, serotype 9V/A is not commonly reported in the Indian setting but has been reported in other countries. ${ }^{10}$

An important observation from the present case series is that all the isolated pneumococcal serotypes were vaccinepreventable. ${ }^{5,6,10}$ This observation is in contrast to earlier reports where non-vaccine-preventable pneumococcal serotypes were also frequently observed. The PCV coverage of pneumococcal serotypes in these studies ranged between 29 and $78 \% .{ }^{3,5,6,9,11}$
Antimicrobial resistance was observed for the following antibiotics: cotrimoxazole, benzylpenicillin, erythromycin, and clindamycin, which is similar to other observations from India. ${ }^{5,6,9,12}$ The majority of the cases were susceptible to all or a few antibiotics, namely, penicillin, ceftriaxone, levofloxacin and cotrimoxazole, vancomycin, and clindamycin. The pneumococcal serotypes with antimicrobial resistance were $19 \mathrm{~F}$ and $19 \mathrm{~A}$, while serotypes 2 and 3 were susceptible to all antibiotics. High susceptibility of serotype 3 toward antimicrobials has been reported in earlier studies. ${ }^{6}$ Resistance to penicillin was low, in line with other Indian studies.,6,12 In the majority, selective or total susceptibility to antimicrobials was observed in the present study. Moreover, the mortality rate was also low; one out of nine cases succumbed to the disease.

Taken together, the present case series delineates the existing antimicrobial usage and other treatment patterns, vaccination coverage and rates, mortality rates, and antimicrobial resistance, along with IPD causative serotypes in Indian children, and emphasizes the need for surveillance studies for monitoring the changes in causative serotypes and antimicrobial resistance patterns in India, given the broad geographic variations in the country.

\section{Conclusion}

Our study is probably one of the first case series of IPDs in immunized children since the availability of PCV in India. This study is an attempt at identifying and serotyping the invasive pneumococcal isolates in our practice, considering the lack of 
serotyping and antibiotic susceptibility studies in India. This study emphasizes the importance of continued surveillance for IPDs and serotyping the pneumococcal isolates to analyze the effects of vaccination on the drug resistance pattern, in particular. The most unique observation in this study is that all of the IPD cases have been caused by vaccine-preventable serotypes. These findings are very encouraging, since, after the inclusion of PCVs in the National Immunization Program, the emergence of non-vaccine serotypes with higher antimicrobial resistance is a matter of grave concern, not only in India but also at the global level.

\section{Clinical Significance}

Invasive pneumococcal disease is a major cause of high mortality in Indian children aged below 5 years. There is an unmet need for regular surveillance of IPD-causing pneumococcal serotypes and antimicrobial resistance patterns among Indian children with IPD. Although pathogen serotyping is uncommon in India, several serotypes have been identified as causative factors for IPD in Indian children, but the emergence of non-vaccine-preventable serotypes is not properly documented. This is probably one of the first case series of IPD in immunized Indian children since the availability of PCV in India, which reveals that pneumococcal serotypes causing IPD in Indian children are largely vaccine-preventable and antibiotic susceptible.

\section{Authorship}

R Kishore Kumar has contributed toward conception, design of the work, acquisition, analysis, interpretation of data, drafting the work and revising, final approval and agreed to be accountable for all aspects of the work in ensuring that questions related to the accuracy or integrity of any part of the work are appropriately investigated and resolved. Sanjay Swamy has contributed toward the collection and follow-up of data and the final approval of the manuscript. Adithi S has contributed toward a collection of data, drafting the initial manuscript, and final approval of the manuscript.

\section{Ethical Clearance}

Not applicable as this is a case series, not a clinical study.

\section{References}

1. Chhatwal J. The unchecked pneumococcal reign over Indian children aged younger than 5 years. Lancet Infect Dis 2017;17(3):243-244. DOI: 10.1016/S1473-3099(16)30543-6.

2. Manoharan A, Jayaraman R. Pneumococcal caccines. Indian J Med Microbiol 2018;36(4):465-474. DOI: 10.4103/ijmm.IJMM_18_442.

3. Manoharan A, Manchanda V, Balasubramanian S, et al. Invasive pneumococcal disease in children aged younger than 5 years in India: a surveillance study. Lancet Infect Dis 2017;17(3):305-312. DOI: 10.1016/S1473-3099(16)30466-2.

4. Shariff M, Choudhary J, Zahoor S, et al. Characterization of Streptococcus pneumoniae isolates from India with special reference to their sequence types. J Infect Dev Ctries 2013;7(02):101-109. DOI: 10.3855/jidc.2553.

5. Balaji V, Jayaraman R, Verghese V, et al. Pneumococcal serotypes associated with invasive disease in under five children in India \& implications for vaccine policy. Indian J Med Res 2015;142(3):286-292. DOI: 10.4103/0971-5916.166588.

6. Molander V, Elisson C, Balaji V, et al. Invasive pneumococcal infections in Vellore, India: clinical characteristics and distribution of serotypes. BMC Infect Dis 2013;13(1):532. DOI: 10.1186/1471-2334-13-532.

7. John TJ, Pai R, Lalitha MK, et al. Prevalence of pneumococcal serotypes in invasive diseases in southern India. Indian J Med Res 1996;104:205-207.

8. Nisarga R, Premalatha R, Shivananda, et al. Hospital-based surveillance of invasive pneumococcal disease and pneumonia in South Bangalore, India. Indian Pediatr 2015;52(3):205-211. DOI: 10.1007/s13312-015-0607-0.

9. Singh J, Sundaresan S, Manoharan A, et al. Serotype distribution and antimicrobial susceptibility pattern in children $\leq 5$ years with invasive pneumococcal disease in India - a systematic review. Vaccine 2017;35(35):4501-4509. DOI: 10.1016/j.vaccine.2017.06.079.

10. Ktari S, Jmal I, Mroua M, et al. Serotype distribution and antibiotic susceptibility of Streptococcus pneumoniae strains in the south of Tunisia: a five-year study (2012-2016) of pediatric and adult populations. Int J Infect Dis 2017;65:110-115. DOI: 10.1016/j. ijid.2017.10.015.

11. John J, Varghese R, Lionell J, et al. Non-vaccine pneumococcal serotypes among children with invasive pneumococcal disease. Indian Pediatr 2018;55(10):874-876. DOI: 10.1007/s13312-018-1398-X.

12. Mathew JL, Patwari AK, Gupta P, et al. Acute respiratory infection and pneumonia in India: a systematic review of literature for advocacy and action: UNICEF-PHFI series on newborn and child health, India. Indian Pediatr 2011;48(3):191-218. DOI: 10.1007/s13312-011-0051-8. 